\title{
REVIEW OF THE ULTRAVIOLET FLUX CALIBRATION
}

\author{
JEFFREY W. KRUK \\ Johns Hopkins University, Baltimore, MD, 21218, USA
}

\section{Introduction}

The practical difficulties in performing laboratory calibrations of instruments at ultraviolet wavelengths are considerably greater than at visible wavelengths, and the concommitant uncertainties are greater as well. In recent years theoretical models of white dwarf atmospheres have been adopted as UV flux standards, with impressive results. In this review, I will discuss the methodology of laboratory flux calibrations in the UV, the internal consistency and potential shortcomings of calibrations based on white dwarf model atmospheres, recent laboratory results, and future prospects.

\section{Methodology}

Absolute calibrations of the sensitivity of ground-based telescopes are performed by direct comparison of the signal observed from a standard star with that of a laboratory standard source (such as a platinum or copper blackbody) located on a nearby mountain top. The major inherent uncertainty is in correcting for the differences in absorption by the atmosphere between the horizontal line of sight to the laboratory blackbody and the vertical line of sight to the star. Different groups have been able to obtain results that are consistent to within about $1.5 \%$. Reviews of these measurements can be found in Megessier (1997) and Hayes (1985).

At UV wavelengths, observations are performed by sounding rockets or by orbiting spacecraft. There is no atmospheric absorption to be corrected, but there is also no means of observing a laboratory flux standard while the instrument is in space. There are no laboratory blackbody sources hot enough to provide a usable flux in the FUV, so electron synchrotrons are the only practical sources whose flux can be calculated from first principles. At present, the only suitable facility for calibration purposes in the US 
is the Synchrotron Ultraviolet Radiation Facility (SURF) at the National Institute for Standards and Technology (NIST). Direct calibration of instruments at SURF is usually impractical, however, and is rarely done. In lieu of direct calibration, instruments are usually calibrated by comparison with secondary (or tertiary) quantum efficiency standards such as photodiodes. Secondary standard photodiodes are typically calibrated by NIST at fixed wavelengths using the continuous spectrum produced by SURF and a double monochromator (the details vary with wavelength; see Canfield \& Swanson 1987). A user's photodiode is then calibrated as a tertiary standard at fixed wavelengths by comparison with the secondary standard photodiodes. The quoted uncertainty in the calibration at this point is typically $8 \%$ in the FUV, and $10-15 \%$ in the EUV. These photodiodes require far more intense photon beams to produce a usable signal than the photon-counting detectors typically used in space astronomy, so the user ordinarily calibrates intermediate reference detectors by comparison with the tertiary standard photodiodes. At this point, the user's instrument can be calibrated by comparison with the intermediate reference detectors. The cumulative uncertainties in the calibration at this point are typically $15 \%$.

\section{Results Prior to Adoption of the White Dwarf Standard}

Prior to 1994, the UV calibration of the International Ultraviolet Explorer (IUE) and the Hubble Space Telescope Faint Object Spectrograph (HST FOS) were based on measurements of the star $\eta$ UMa obtained by a series of rocket flights and the OAO-2 satellite (which in turn was calibrated by rocket flights). These sounding rocket instruments were calibrated using NIST photodiodes as described above, and the measurements of $\eta \mathrm{UMa}$ were consistent to within about 10\% (see Bohlin et al. (1980) and references therein). When spectra obtained with IUE of numerous hot DA white dwarf stars were compared with theoretical predictions, similar wavelengthdependent discrepancies of $10-15 \%$ were observed in each case. Similar discrepancies between observation and expectation were also obtained for other hot stars and such dissimilar sources as BL-Lac objects and quasars. The uncertainties in the model atmosphere predictions for hot DA's were expected to be about $1 \%$ relative to the observed fluxes at visible wavelengths, so these discrepancies ultimately led to the abandonment of the laboratory-based calibration and the adoption of white dwarf models as calibration standards in the UV.

Prior to the Hopkins Ultraviolet Telescope (HUT) and Orbiting Retreivable Far and Extreme Ultraviolet Spectrograph (ORFEUS) space shuttle flights, the only spectral data available below $1150 \AA$ with absolute fluxes were obtained with the Voyager UVS or by sounding rocket instruments. 
Spectra obtained by the rocket instruments were usually consistent with the corresponding Voyager spectra to within their measurement uncertainties (typically $15 \%$ ) longward of $1200 \AA$, but discrepancies sometimes grew dramatically at shorter wavelengths, ranging from factors of two to as much as seven over $950-1000 \AA$. The Voyager calibration is described in Holberg et al. (1982), and a summary of the controversies at short wavelengths can be found in Holberg et al. (1991).

\section{White Dwarf Models}

In 1994 the HST FOS switched to defining the UV sensitivity based on theoretical models of DA white dwarf atmospheres. Initially the white dwarf G191-B2B was used for this purpose; subsequently a combination of stars of widely varying effective temperatures was employed: G191-B2B $(61,300 \mathrm{~K}), \mathrm{HZ} 43(50,000 \mathrm{~K}), \mathrm{GD} 153(38,500 \mathrm{~K})$, and GD $71(32,300 \mathrm{~K})$. Fluxes for these stars were calculated by D.Finley using the model code of D. Koester. Effective temperatures and gravities for each star were determined from fits to Balmer line profiles, and the models were normalized to match Landolt V photometry (Finley et al., 1997). These four independent models provide internally consistent results: when using this calibration the spectrum of each of these four stars agreed with its theoretical model flux to within $2 \%$ over the full FOS wavelength range of $1150-8000 \AA$ (Bohlin et al., 1995).

The final archive of IUE low dispersion spectra was reprocessed with a flux calibration defined by a model for G191-B2B (Nichols \& Linsky, 1996). The model code used was the same as for the FOS, but the effective temperature was slightly lower, and the normalization was chosen so that fluxes for the brightest IUE standard stars matched the corresponding OAO-2 fluxes over $2100-2300 \AA$. The difference in $T_{\text {eff }}(58,000 \mathrm{~K} v s .61,300 \mathrm{~K})$ causes the IUE fluxes at Ly $\alpha$ to be about $2 \%$ lower than the FOS relative to the visible, and the different normalization causes IUE fluxes to be $6 \%$ lower than the FOS for the same star.

The internal consistency of white dwarf models down to $912 \AA$ can be tested by HUT spectra obtained during the Astro- 2 mission. HUT provides spectrophotometry over $820-1840 \AA$ at approximately $3 \AA$ resolution (Davidsen et al., 1992). In addition to the four FOS standard DA stars (which have surface gravities in the range of 7.7-8.0), HUT also observed GD $50(41,000 \mathrm{~K}, \log \mathrm{g}=9.0), \mathrm{RE} 0512-004(32,000 \mathrm{~K}, \log \mathrm{g}=7.4)$, and Wolf $1346(20,000 \mathrm{~K}, \log \mathrm{g}=8.0)$. The final calibration was defined by first computing the effective area from comparisons of the observed spectrum with model atmosphere predictions separately for each of the stars HZ 43, GD 153, and GD 71, and then averaging the results. The models are the 
same as used by the FOS. For all 7 stars, the resulting observed flux agrees with the predicted flux to within $5 \%$ (usually much better) over the entire wavelength range of $912 \AA-1840 \AA$, despite the very wide range of effective temperatures and surface gravities. This is particularly noteworthy, since the flux at the Lyman edge is a very sensitive function of the effective temperature. The star Wolf 1346 is cool enough that opacity arising from quasi-molecular states of Ly $\beta$ had to be added to the model code (Koester et al., 1996). Further information on the Astro-2 HUT calibration can be found in Kruk et al. 1995.

\section{EUV Calibration}

Models of white dwarfs cannot be used as flux standards at EUV wavelengths because there is no independent means of determining the opacity of interstellar H I. The Extreme Ultraviolet Explorer (EUVE) is therefore using its preflight laboratory calibration, which has uncertainties of $15 \%$ $20 \%$. The instrument sensitivity has been stable since launch, and the its observed fluxes have been consistent with the few other measured fluxes that exist. The biggest systematic error in EUVE fluxes arises from overlapping diffraction orders longward of $560 \AA$ (Dupuis et al., 1995).

\section{New Laboratory Calibration}

Following the first flight of HUT on the space shuttle Columbia in December 1990 , we were able to perform a thorough post-flight calibration. This calibration differed from previous UV radiometric calibrations in that we were able to calibrate the spectrograph throughput directly at SURF, thereby avoiding buildup of uncertainties through the long sequence of transfer standards. The primary mirror reflectivity was measured in the calibration facilities at Johns Hopkins, since the instrument as a whole was too large to install in the vacuum chamber at SURF. The ratio of the white dwarf derived effective area to the laboratory effective area had a mean value of 1.0044 over the wavelength range $912-1840 \AA$, a slope of $7.61 \times 10^{-6} \AA^{-1}$ (which corresponds to a relative difference of only $0.35 \%$ at either end of the spectrum), and fluctuations about the mean with an RMS amplitude of $3 \%$. The uncertainties in the laboratory calibration are: $4 \%$ for the overall normalization, $5 \%$ relative to the mean on scales of $50-100 \AA$, and an uncertainty in the overall slope that corresponds to $1 \%$ at $1840 \AA$ and which increases smoothly to $3 \%$ at $912 \AA$. This calibration is described in detail by Kruk et al. (1997).

Astro-1 HUT spectra of 5 hot stars were compared with the corresponding Voyager fluxes. When averaged over broad bands, the spectra agreed to 
within $10 \%$. Given the absolute laboratory calibration of HUT, this should help resolve the controversies over the Voyager flux measurements.

The uncertainties in this laboratory calibration are not that much larger than the intrinsic uncertainties in the white dwarf calibration at wavelengths shortward of Ly $\alpha$. The combined uncertainties in the flux of Vega and the $\mathrm{V}$-band photometry give an absolute normalization uncertainty of about $2 \%$. The internal uncertainties in the effective temperature for a well-studied star such as G191-B2B are typically about $300 \mathrm{~K}$, leading to negligible uncertainties in the FUV flux. External uncertainties, such as different treatments in pressure broadening when fitting the line profiles, do lead to significant uncertainties in the FUV flux. For G191-B2B, these uncertainties in the predicted flux are estimated to be $1 \%$ at $1840 \AA, 2.3 \%$ at $1000 \AA$, and almost $7 \%$ at the Lyman edge. The uncertainties in the surface gravity have little effect on the continuum flux, but do affect the Lyman line profiles.

An additional intrinsic source of uncertainty in the theoretical predicted flux arises from the treatment of Stark broadening of the Lyman lines. At present, the method of Schöning \& Butler (1989) is used to calculate the Stark-broadened Lyman and Balmer line profiles. This is based on the theory of Vidal et al. (1970), which makes the assumption that the individual lines do not overlap. This assumption is badly violated in the high gravity atmosphere of a white dwarf. An empirical correction for this effect is incorporated into the models for calculating Balmer line profiles (Bergeron, 1993). The same correction is also used for the Lyman lines, but the only justification for doing so is that it permits an internally consistent fit to the HUT spectra. The lack of a proper theory of Stark broadening is the major shortcoming in models of hot DA white dwarfs at present.

\section{Modelling of metals in G191-B2B}

The star G191-B2B is a potentially poor choice as a calibration standard, because of the presence of trace quantities of heavy elements in its photosphere. Despite the low abundances, these elements cause strong line blanketing shortward of $250 \AA$ (Dupuis et al., 1995), which in turn causes backwarming of the atmosphere and an apparent increase in the flux longward of 240 A. The stars HZ 43, GD 71, and GD 153 were also measured by Dupuis et al. and were found to have atmospheres that were truly pure hydrogen. The internal consistency of the HST FOS and Astro-2 HUT calibrations, and the laboratory calibration of the Astro-1 HUT sensitivity all indicate that using a pure hydrogen model to fit the Balmer lines of G191-B2B does result in a model that accurately predicts the flux at FUV wavelengths. However, it was only very recently that a theoretical model 
was able to predict both the EUV spectrum of G191-B2B and the UVvisible spectrum simultaneously (Lanz et al., 1996). While there is still some difficulty with detailed fits to the EUV He lines, the model of Lanz et al. is an important advance, not only in the state of the art in modelling techniques, but also for validating the previously adopted flux of G191-B2B at FUV wavelengths for use as a flux standard.

\section{Prospects for the Future}

The techniques used to calibrate HUT at SURF could certainly be improved upon. A further factor of two reduction in the ultimate laboratory incertainties seems feasible, especially in light of planned upgrades to the SURF accelerator. However, I am not aware of any plans for a suitable sounding rocket or other retrievable spectroscopic instrument that could be so calibrated and used to test white dwarf models. The most likely opportunity will be with a sounding rocket. However, given the limited possible aperture and the short duration of a sounding rocket flight, it might be prefereable to observe a hot sdO star rather than a white dwarf in order to obtain a high signal to noise spectrum. Such a star could then serve as a standard, much as Vega is used in the visible.

\section{References}

Bergeron, P. 1993, in White Dwarfs: Advances in Observation and Theory, NATO ASI Series, ed. M.A. Barstow (Kluwer: Dordrecht), p. 267

Bohlin, R., Holm, A., Savage, B., Snijders, M., \& Sparks, W. 1980, A\&A 85, 1

Bohlin, R.C., Colina, L., \& Finley, D.S. 1995, AJ 110, 1316

Canfield, L.R., \& Swanson, N. 1987, J. Res. NBS 92, 97

Davidsen, A.F., Long, K.S., Durrance, S.T., Blair, W.P., Bowers, C.W., Conard, S.J., Feldman, P.D., Ferguson, H.C., Fountain, G.H., Kimble, R.A., Kriss, G.A., Moos, H.W., Potocki, K.A., 1992, ApJ 392264

Dupuis, J., Vennes, S., Bowyer, S., Pradhan, A.K., \& Thejll, P. 1995, ApJ 455, 574

Finley, D.S., Koester, D., \& Basri, G. 1997, ApJ, submitted

Hayes, D.S. 1985, in Calibration of Fundamental Stellar Quantities, Proc. IAU Symp. No. 111, eds. D. Hayes, L. Pasinetti, \& A. Phillip (Reidel, Dordrecht), p. 225

Holberg, J.B., Forrester, W.T., Shemansky, D.E., \& Barry, D.C. 1982, ApJ 257, 656

Holberg, J.B., Ali, B., Carone, T.E., \& Polidan, R.S. 1991, ApJ 375, 716

Koester, D., Finley, D.S., Allard, N.F., Kruk, J.W., \& Kimble, R.A. 1996, ApJ 463, L93 Kruk, J.W., Durrance, S.T., Kriss, G.A., Davidsen, A.F., Blair, W.P., Espey, B.R., \& Finley, D.S. 1995, ApJ 454, L1

Kruk, J.W., Kimble, R.A., Buss, R.H., Davidsen, A.F., Durrance, S.T., Finley, D.S., Holberg, J.B., \& Kriss, G.A. 1997, ApJ 482, in press

Lanz, T., Barstow, M.A., Hubeny, I., \& Holberg, J.B. 1996, ApJ 473, 1089

Megessier, C., these proceedings

Nichols, J.S., \& Linsky, J.L. 1996, AJ 111, 517

Schoning, T., \& Butler, K. 1989, A\&AS 78, 51

Vidal, C.R., Cooper, J., \& Smith, E.W. 1970, ApJS 25, 37

Discussion of this paper appears at the end of these Proceedings. 